\title{
Pregnancy Differentially Regulates the Collagens Types I and III in Left Ventricle from Rat Heart
}

\author{
Sarai Limon-Miranda, ${ }^{1}$ Diana G. Salazar-Enriquez, ${ }^{2}$ \\ Jesus Muñiz, ${ }^{2}$ Mario V. Ramirez-Archila, ${ }^{2}$ Enrique A. Sanchez-Pastor, ${ }^{2}$ Felipa Andrade, ${ }^{3}$ \\ Jose G. Soñanez-Organis, ${ }^{1}$ Edgar F. Moran-Palacio, ${ }^{1}$ and Adolfo Virgen-Ortiz ${ }^{1}$ \\ ${ }^{1}$ Division of Science and Engineering, Department of Chemical Biological and Agropecuary Sciences, University of Sonora, \\ Lazaro Cardenas 100, Colonia, Campus Navojoa, Francisco Villa, 85890 Navojoa, SON, Mexico \\ ${ }^{2}$ University Center for Biomedical Investigations, University of Colima, 28045 Colima, COL, Mexico \\ ${ }^{3}$ Technological Institute of Colima, 28976 Villa de Alvarez, COL, Mexico
}

Correspondence should be addressed to Adolfo Virgen-Ortiz; avirgen@navojoa.uson.mx

Received 26 February 2014; Revised 9 July 2014; Accepted 10 July 2014; Published 24 July 2014

Academic Editor: Andre Van Wijnen

Copyright (C) 2014 Sarai Limon-Miranda et al. This is an open access article distributed under the Creative Commons Attribution License, which permits unrestricted use, distribution, and reproduction in any medium, provided the original work is properly cited.

\begin{abstract}
The pathologic cardiac remodeling has been widely documented; however, the physiological cardiac remodeling induced by pregnancy and its reversion in postpartum are poorly understood. In the present study we investigated the changes in collagen I (Col I) and collagen III (Col III) mRNA and protein levels in left ventricle from rat heart during pregnancy and postpartum. Col I and Col III mRNA expression in left ventricle samples during pregnancy and postpartum were analyzed by using quantitative PCR. Data obtained from gene expression show that Col I and Col III in left ventricle are upregulated during pregnancy with reversion in postpartum. In contrast to gene expression, the protein expression evaluated by western blot showed that Col I is downregulated and Col III is upregulated in left ventricle during pregnancy. In conclusion, the pregnancy differentially regulates collagens types I and III in heart; this finding could be an important molecular mechanism that regulates the ventricular stiffness in response to blood volume overload present during pregnancy which is reversed in postpartum.
\end{abstract}

\section{Introduction}

Cardiovascular and hormonal changes are induced during pregnancy, which include a marked increase in cardiac output, heart rate, blood volume, and stroke volume and a decrease in total vascular resistance [1-3]. In rats, cardiomyocytes are undergoing a metabolic remodeling in late pregnancy [4]. In human and animals (rats and mice), it has been reported that a prolonged volume overload during pregnancy triggers eccentric cardiac hypertrophy which is reversible in postpartum. This hypertrophy is the result of individual growth of cardiac myocytes, predominantly in the longitudinal axis $[5,6]$. The molecular signaling pathways in pathological cardiac hypertrophy and cardiac remodeling have been widely studied; however, the physiological cardiac remodeling induced by pregnancy and the reversion in postpartum are poorly understood.
The molecular signaling implicated in pregnancy-induced cardiac hypertrophy has been studied by different authors [5, 7-10]; however, there is little information about the remodeling of cardiac extracellular matrix during pregnancy. The extracellular matrix is a fundamental structure for cardiac function and is composed mainly of collagen, which contributes to passive tension in stretched cardiac muscle, thereby influencing the end-diastolic volume of the heart $[11,12]$. The cardiac stiffness is associated to the expression of collagen isoforms, mostly type I collagen (Col I) and type III collagen (Col III) [13]. Col I is well-structured fibers that resist extension, while Col III forms a fine reticular network that is more compliant [14]. In clinical studies, the increase in both collagen content and Col I/Col III ratio leads to reduced myocardial compliance and diastolic dysfunction, which can result in heart failure $[15,16]$. Despite its clinical importance, the adaptive remodeling in collagen 
isoforms expression in heart during pregnancy has not been elucidated.

In the present study, we investigated changes in Col I and Col III mRNA and protein during pregnancy and postpartum in rat heart. Our data could help to understand the molecular mechanisms involved in the remodeling of cardiac extracellular matrix during pregnancy and postpartum.

\section{Materials and Methods}

2.1. Animals. Animal care and experimental procedures were approved by the Ethics Committee of the University of Colima, using guidelines based on the Guide for the Care and Use of Laboratory Animals (US Department of Health, $\mathrm{NIH}$ ). Three-month-old female Sprague-Dawley rats were separated into four groups: controls or nonpregnant rats (NP, diestrus $260 \pm 8 \mathrm{~g}, n=10$ ), midpregnancy rats (MP, 12 days of gestation, $290 \pm 10 \mathrm{~g}$ ), late-pregnancy rats (LP, 21 days of gestation, $330 \pm 10 \mathrm{~g}, n=10$ ), and postpartum rats (PP, 7 days, $270 \pm 8 \mathrm{~g}, n=10$ ). All rats were provided with water and food ad libitum and were maintained in individual acrylic cages on a $12: 12 \mathrm{~h}$ light-dark cycle with an average daily temperature of $24 \pm 1^{\circ} \mathrm{C}$ and an average humidity of $60-70 \%$. The day of the experiment, all the rats, previously anesthetized (pentobarbital sodium, $50 \mathrm{mg} / \mathrm{kg}$, intraperitoneally), were sacrificed by cervical dislocation and then the hearts were excised and weighed; subsequently the left ventricle was dissected, immediately frozen in liquid nitrogen, and stored at $-80^{\circ} \mathrm{C}$ for posterior analysis.

2.2. Quantitative PCR. Total RNA was isolated individually from frozen left and right ventricle samples using TRIzol reagent (Invitrogen) following the manufacturer's instructions. Genomic DNA in total RNA was eliminated by digestion using recombinant DNase I (Roche, Indianapolis, IN, USA) as specified by the manufacturer. Separate cDNAs from each tissue were synthesized from total DNA-free RNA $(1 \mu \mathrm{g})$ using the QuantiTect Reverse Transcription kit (Qiagen, Valencia, CA, USA) and oligo-dT (0.5 $\mu \mathrm{g})$.

Col I, Col III, and glyceraldehyde 3-phosphate dehydrogenase (GAPDH) mRNA were measured by quantitative PCR (qPCR) using the primers shown in Table 1. qPCR reactions were performed for Col I and Col III and normalized by the mRNA expression of GAPDH using $3 \mu \mathrm{L}$ of cDNA for each gene. Standard curves of Col I, Col III, and GAPDH were run to determine the efficiency of amplification using dilutions from $5 E 10^{-4}$ to $5 E 10^{-8} \mathrm{ng} / \mu \mathrm{L}$ of PCR fragment. For each measurement, the expression levels $(\mathrm{ng} / \mu \mathrm{L})$ were normalized to GAPDH and expressed as the ratio of Col I or Col III to GAPDH. GAPDH was used as a housekeeping gene; previous studies demonstrated that GAPDH expression in cardiac tissues did not change during pregnancy $[5,17]$.

2.3. Western-Blot Analysis. To evaluate protein expression levels, left ventricular tissue was homogenized with a Polytron homogenizer in lysis buffer and protease inhibitor
TABLE 1: Primers used for quantitative PCR analysis.

\begin{tabular}{ll}
\hline Gene/primer name & Primer sequence $\left(5^{\prime}-3^{\prime}\right)$ \\
\hline $\begin{array}{l}\text { Collagen I (Col I) } \\
\text { Col I-forward } \\
\text { Col I-reverse }\end{array}$ & AAGACATCCCTGAAGTCAGC \\
Collagen III (Col III) & CCTATGACTTCTGCGTCTGG \\
Col III-forward & ACAGCAGTCCAATGTAGATG \\
Col III-reverse & GAGCAGGTGTAGAAGGCTG \\
$\begin{array}{l}\text { Glyceraldehyde-3-phosphate } \\
\text { dehydrogenase (GAPDH) } \\
\text { GAPDH-forward }\end{array}$ & \\
GAPDH-reverse & GGACATTGTTGCCATCAACG \\
\hline
\end{tabular}

cocktail (Roche). Fifty micrograms of total protein was separated by $10 \%$ SDS-PAGE. Separated proteins were electrotransferred to nitrocellulose membranes. Membranes were blocked with 5\% blocking agent (Amersham, GE healthcare) and immunoblotted using the following antibodies: Col I and Col III, and GAPDH was used as a load control (Santa Cruz Biotechnology). After that, a secondary horseradish peroxidase-conjugated antibody was applied for 1 hour at room temperature. The blots were developed with a chemiluminescence detection system (Amersham, GE Heath care) and visualized by exposure to Kodak radiographic film. Density of bands was measured with Image J (National Institutes of Health, Bethesda, MD, USA).

2.4. Statistical Analysis. The obtained data were expressed as means \pm SEM. To compare two groups Student's $t$-test was used. For comparison of more than two experimental groups, analysis of variance of one factor and post hoc Tukey's test were applied. All the differences were considered statistically significant with a $P \leq 0.05$. Statistical analysis was conducted with the software Minitab Release version 12.

\section{Results and Discussion}

Col I and Col III mRNA and protein expression levels were quantified in left ventricle from rat heart to determinate their expression in response to pregnancy and postpartum. Col I and Col III transcripts increased $(P>0.05)$ 8.2-fold and 8.8fold, respectively, after 12 days of pregnancy (see Figure 1). In contrast, after 21 days of pregnancy Col I increased $(P>0.05)$ 2.7-fold and Col III increased 10.9-fold compared to nonpregnant group. In postpartum, Col I and Col III transcripts returned to basal levels (see Figure 1).

On the other hand, the protein expression analysis revealed that Col I expression in left ventricle decreased significantly $(P>0.05)$ during pregnancy (MP and LP groups) and postpartum compared with nonpregnant group (see Figures 2 and 3). Moreover, the results of Col III expression analysis in left ventricle show that, at protein level, an increase $(P>0.05)$ during pregnancy reaching a maximal point of expression in late pregnancy is observed (LP group increased 1.6-fold versus NP group; see Figure 3). 

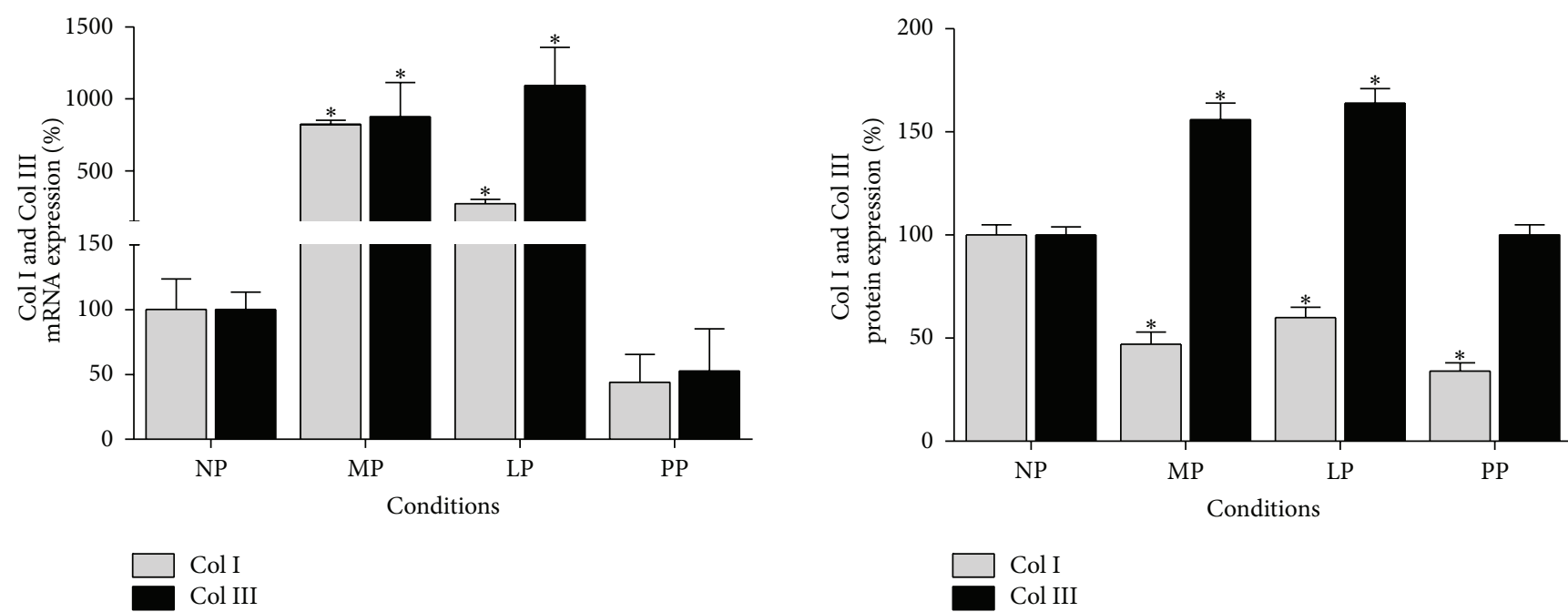

FIGURE 1: Collagen isoforms mRNA expression in left ventricle during pregnancy and postpartum. NP: nonpregnant group $(n=$ 10), MP: midpregnancy group $(n=10)$, LP: late-pregnancy group $(n=10)$, and PP: postpartum group $(n=10)$. Values are expressed as means \pm SEM. ${ }^{*} P<0.05$ versus $\mathrm{NP}$ group.

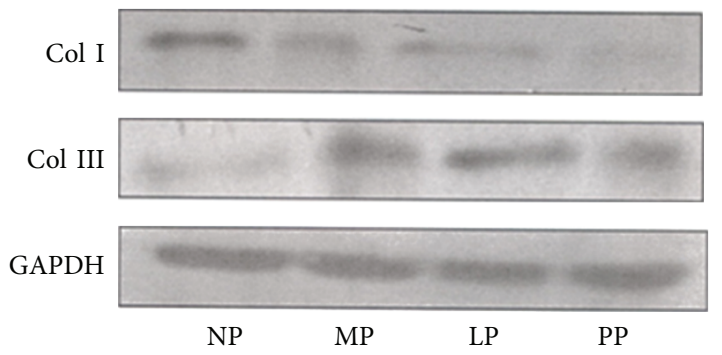

FIGURE 2: Representative immunoblots. NP: nonpregnant group; MP: midpregnancy group; LP: late-pregnancy group; and PP: postpartum group.

The data obtained in this study also show that Col III expression level is similar in nonpregnant and postpartum group (see Figure 3).

Collagens are the main component of cardiac extracellular matrix, which participate in the pathologic heart remodeling [18-21]. However, the Col I and Col III participation in heart remodeling in response to pregnancy is not well defined. In the present study, the Col I and Col III mRNA and protein expression levels in left ventricle from rat heart were determined during pregnancy. Col I and Col III mRNA expression levels are upregulated in left ventricle during pregnancy and reversed during postpartum. The increase in Col I and Col 111 mRNA along with differential protein expression suggests that both collagens contribute to heart remodeling during pregnancy. A previous study reported that collagen isoforms gene expression in cardiac tissue did not change during pregnancy [22], while other reports showed that Col III is downregulated in late pregnancy [23]. However, our data clearly show that Col I and Col III mRNA expression levels in left ventricle increased at 12 and 21 days of pregnancy.

Also, we show that Col III protein expression in left ventricle increased in late pregnancy while Col I decreased. Some reports have shown that matrix metalloproteinase-2 in heart, which cleaves Col III [24], is downregulated during late pregnancy [25]; this could suggest that a suppression in matrix metalloproteinase-2 expression in heart promotes Col III expression in extracellular matrix. In contrast, other studies using histological analysis have reported a minimal increase in collagen content in the heart during pregnancy [9] or no difference [10, 22].

Therefore, our data suggest that the differential expression of collagen isoforms in heart during pregnancy could play an important role in the regulation of the developed passive tension in myocardial walls during the diastolic filling. The changes in collagen isoforms expression observed in this work could explain the decrease in cardiac stiffness in late pregnancy reported in previous studies [6], suggesting an adaptive mechanism to compensate the demand of blood volume overload in heart during pregnancy.

The mechanism that regulates the collagen expression in heart during pregnancy is still unknown. An early study reported that estrogens reduce the $\mathrm{Col}$ I/Col III ratio in age-related left ventricular remodeling; this reduction was result of a decrease in Col I accompanied by an increase in Col III protein expression [26]. It is known that during pregnancy there are characteristic changes in hormonal levels and in early pregnancy the level of progesterone is increased while the level of estrogens is increased in late pregnancy [27]. These changes could be regulating collagen expression, and therefore the mechanism implicated in the control of differential expression of collagen isoforms during pregnancy could be hormonal. Recent studies have shown that, in early pregnancy, progesterone regulates development of cardiac hypertrophy through a calcineurin-dependent pathway [17] 
and the activation of ERK1/2 [10]. Moreover, estrogens increase MCIP1 (modulatory calcineurin interacting protein 1) expression (a calcineurin inhibitor) [17] suggesting that estrogens inhibit the effect of calcineurin in late pregnancy. However, more experiments should be performed to demonstrate that estrogens and progesterone are also involved in the regulation of the expression of collagen isoforms in cardiac remodeling during pregnancy. Besides, although Col I and Col III mRNA were upregulated in left ventricle; interestingly at protein level, they were differentially expressed in heart during pregnancy, leaving to elucidate the mechanism of posttranscriptional regulation that is involved. Recent reports show that microRNAs (miRNAs) play an important role in the regulation of cardiovascular diseases [28-31]. The miRNAs miR-29 family and miR-133 regulate mRNAs that encode proteins involved in fibrosis during the pathologic cardiac remodeling [32, 33]; however, the participation of these miRNAs in physiologic cardiac remodeling induced by pregnancy is still unknown.

\section{Conclusions}

Collagen is regulated in the physiological cardiac remodeling induced by pregnancy and postpartum. The differential expression of collagen at protein level in left ventricle can act as an important regulation mechanism in the blood volume overload that is present during pregnancy and that is reversed in postpartum.

\section{Conflict of Interests}

The authors declare that there is no conflict of interests regarding the publication of this paper.

\section{Acknowledgments}

This study was supported by Mexico's National Council for Science and Technology to Ph.D. Adolfo Virgen-Ortiz, Project CB-155238. The authors would like to thank Biol. Victor M. Gutierrez Garcia for his technical assistance in handling animals.

\section{References}

[1] L. A. Simmons, A. G. Gillin, and R. W. Jeremy, "Structural and functional changes in left ventricle during normotensive and preeclamptic pregnancy," The American Journal of PhysiologyHeart and Circulatory Physiology, vol. 283, no. 4, pp. H1627H1633, 2002.

[2] A. Y. Wong, S. Kulandavelu, K. J. Whiteley, D. Qu, B. L. Langille, and S. L. Adamson, "Maternal cardiovascular changes during postpartum in mice," American Journal of Physiology-Heart and Circulatory Physiology, vol. 282, pp. H918-H925, 2002.

[3] O. Savu, R. Jurcuţ, S. Giuşcă et al., "Morphological and functional adaptation of the maternal heart during pregnancy," Circulation: Cardiovascular Imaging, vol. 5, pp. 289-297, 2012.

[4] V. Bassien-Capsa, F. M. Elzwiei, S. Aneba, J. C. Fouron, B. Comte, and A. Chorvatova, "Metabolic remodelling of cardiac myocytes during pregnancy: the role of mineralocorticoids," Canadian Journal of Cardiology, vol. 27, no. 6, pp. 834-842, 2011.

[5] M. Eghbali, R. Deva, A. Alioua et al., "Molecular and functional signature of heart hypertrophy during pregnancy," Circulation Research, vol. 96, no. 11, pp. 1208-1216, 2005.

[6] A. Virgen-Ortiz, J. L. Marin, A. Elizalde et al., "Passive mechanical properties of cardiac tissues in heart hypertrophy during pregnancy," Journal of Physiological Sciences, vol. 59, no. 5, pp. 391-396, 2009.

[7] A. M. D. Gonzalez, J. C. Osorio, C. Manlhiot, D. Gruber, S. Homma, and S. Mital, "Hypertrophy signaling during peripartum cardiac remodeling," The American Journal of Physiology, vol. 293, no. 5, pp. H3008-H3013, 2007.

[8] P. Fischer and D. Hilfiker-Kleiner, "Survival pathways in hypertrophy and heart failure: the gp130-STAT3 axis," Basic Research in Cardiology, vol. 102, no. 5, pp. 393-411, 2007.

[9] K. Lemmens, K. Doggen, and G. W. de Keulenaer, "Activation of the neuregulin/ErbB system during physiological ventricular remodeling in pregnancy," The American Journal of Physiology-Heart and Circulatory Physiology, vol. 300, no. 3, pp. H931-H942, 2011.

[10] E. Chung, F. Yeung, and L. A. Leinwand, "Akt and MAPK signaling mediate pregnancy-induced cardiac adaptation," Journal of Applied Physiology, vol. 112, no. 9, pp. 1564-1575, 2012.

[11] H. L. Granzier and T. C. Irving, "Passive tension in cardiac muscle: contribution of collagen, titin, microtubules, and intermediate filaments," Biophysical Journal, vol. 68, no. 3, pp. 10271044, 1995.

[12] Y. Wu, O. Cazorla, D. Labeit, S. Labeit, and H. Granzier, "Changes in titin and collagen underlie diastolic of cardiac muscle," Journal of Molecular and Cellular Cardiology, vol. 32, pp. 2151-2162, 2000.

[13] M. Pauschinger, D. Knopf, S. Petschauer et al., "Dilated cardiomyopathy is associated with significant changes in collagen type I/III ratio," Circulation, vol. 99, no. 21, pp. 2750-2756, 1999.

[14] I. Medugorac, "Characterization of intramuscular collagen in the mammalian left ventricle," Basic Research in Cardiology, vol. 77, no. 6, pp. 589-598, 1982.

[15] K. T. Weber, J. E. Jalil, J. S. Janicki, and R. Pick, "Myocardial collagen remodeling in pressure overload hypertrophy. A case for interstitial heart disease," American Journal of Hypertension, vol. 2, pp. 931-940, 1989.

[16] M. M. H. Marijianowski, P. Teeling, J. Mann, and A. E. Becker, "Dilated cardiomyopathy is associated with an increase in the type I/type III collagen ratio: a quantitative assessment," Journal of the American College of Cardiology, vol. 25, no. 6, pp. 12631272, 1995.

[17] E. Chung, F. Yeung, and L. A. Leinwand, "Calcineurin activity is required for cardiac remodelling in pregnancy," Cardiovascular Research, vol. 100, no. 3, pp. 402-410, 2013.

[18] J. N. Cohn, R. Ferrari, and N. Sharpe, "Cardiac remodelingconcepts and clinical implications: a consensus paper from an International Forum on Cardiac Remodeling," Journal of the American College of Cardiology, vol. 35, no. 3, pp. 569-582, 2000.

[19] J. Díez, A. González, B. López, and R. Querejeta, "Mechanisms of disease: pathologic structural remodeling is more than adaptive hypertrophy in hypertensive heart disease," Nature Clinical Practice Cardiovascular Medicine, vol. 2, pp. 209-216, 2005.

[20] D. Fan, A. Takawale, J. Lee, and Z. Kassiri, "Cardiac fibroblasts, fibrosis and extracellular matrix remodeling in heart disease," Fibrogenesis and Tissue Repair, vol. 5, pp. 1-13, 2012. 
[21] K. T. Weber, Y. Sun, S. K. Bhattacharya, R. A. Ahokas, and I. C. Gerling, "Myofibroblast-mediated mechanisms of pathological remodelling of the heart," Nature Reviews Cardiology, vol. 10, pp. 15-26, 2013.

[22] M. B. Aljabri, N. T. Songstad, T. Lund et al., "Pregnancy protects against antiangiogenic and fibrogenic effects of angiotensin II in rat hearts," Acta Physiologica, vol. 201, pp. 445-456, 2011.

[23] E. Chung, J. Heimiller, and L. A. Leinwand, "Distinct cardiac transcriptional profiles defining pregnancy and exercise," PLoS ONE, vol. 7, no. 7, Article ID e42297, 2012.

[24] B. Steffensen, U. M. Wallon, and C. M. Overall, "Extracellular matrix binding properties of recombinant fibronectin type IIlike modules of human $72-\mathrm{kDa}$ gelatinase/type IV collagenase. High affinity binding to native type I collagen but not native type IV collagen," The Journal of Biological Chemistry, vol. 270, no. 19, pp. 11555-11566, 1995.

[25] S. Umar, R. Nadadur, A. Iorga, M. Amjedi, H. Matori, and M. Eghbali, "Cardiac structural and hemodynamic changes associated with physiological heart hypertrophy of pregnancy are reversed postpartum," Journal of Applied Physiology, vol. 113, pp. 1253-1259, 2012.

[26] Y. Xu, I. A. Arenas, S. J. Armstrong, and S. T. Davidge, "Estrogen modulation of left ventricular remodeling in the aged heart," Cardiovascular Research, vol. 57, no. 2, pp. 388-394, 2003.

[27] P. S. Lapolt, D. W. Matt, H. L. Judd, and J. K. Lu, "The relation of ovarian steroid levels in young female rats to subsequent estrous cyclicity and reproductive function during aging," Biology of Reproduction, vol. 35, pp. 1131-1139, 1986.

[28] A. Carè, D. Catalucci, F. Felicetti et al., "MicroRNA-133 controls cardiac hypertrophy," Nature Medicine, vol. 13, no. 5, pp. 613618, 2007.

[29] T. Thum, P. Galuppo, C. Wolf et al., "MicroRNAs in the human heart: a clue to fetal gene reprogramming in heart failure," Circulation, vol. 116, no. 3, pp. 258-267, 2007.

[30] E. Van Rooij and E. N. Olson, "Searching for miR-aclesin cardiac fibrosis," Circulation Research, vol. 104, no. 2, pp. 138140, 2009.

[31] D.-L. Dong and B.-F. Yang, "Role of microRNAs in cardiac hypertrophy, myocardial fibrosis and heart failure," Acta Pharmaceutica Sinica B, vol. 1, no. 1, pp. 1-7, 2011.

[32] E. Van Rooij, L. B. Sutherland, J. E. Thatcher et al., "Dysregulation of microRNAs after myocardial infarction reveals a role of miR-29 in cardiac fibrosis," Proceedings of the National Academy of Sciences of the United States of America, vol. 105, no. 35, pp. 13027-13032, 2008.

[33] S. Chen, P. Puthanveetil, B. Feng, S. J. Matkovich, G. W. Dorn II, and S. Chakrabarti, "Cardiac miR-133a overexpression prevents early cardiac fibrosis in diabetes," Journal of Cellular and Molecular Medicine, vol. 18, no. 3, pp. 415-421, 2014. 


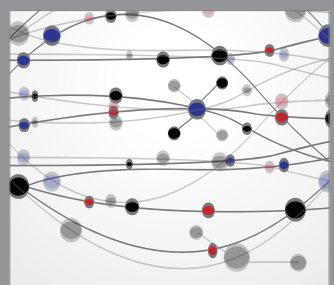

The Scientific World Journal
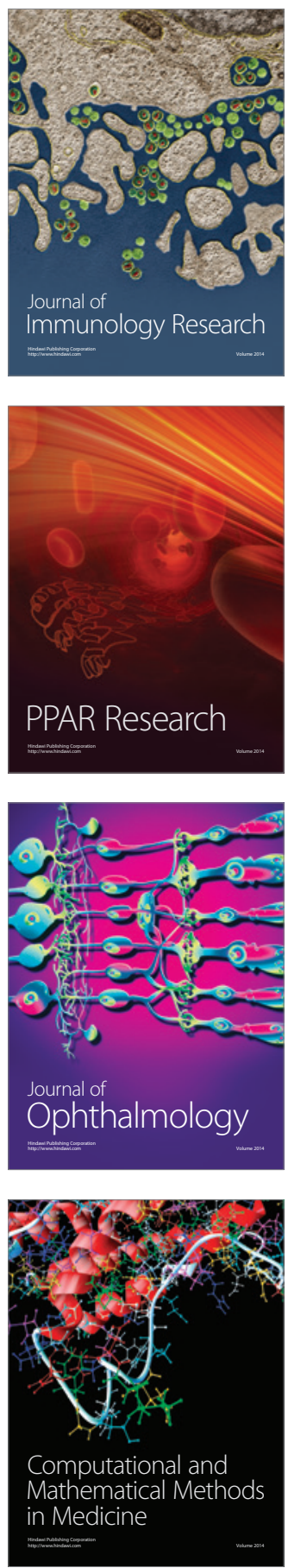

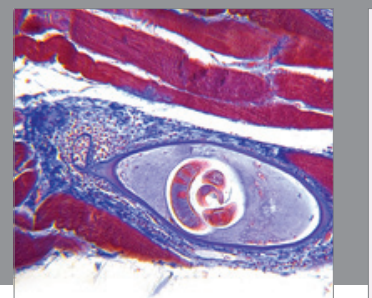

Gastroenterology

Research and Practice
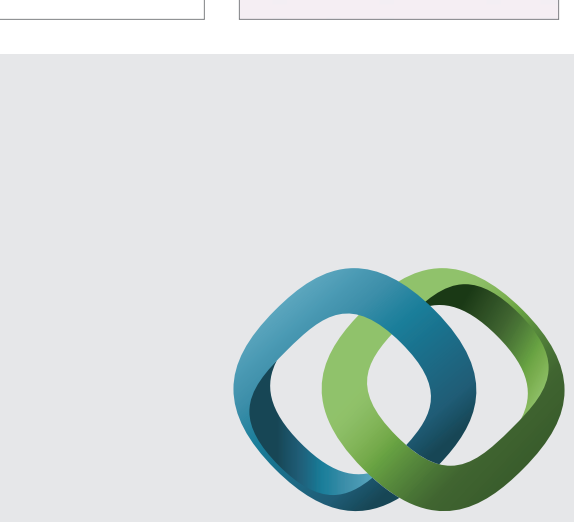

\section{Hindawi}

Submit your manuscripts at

http://www.hindawi.com
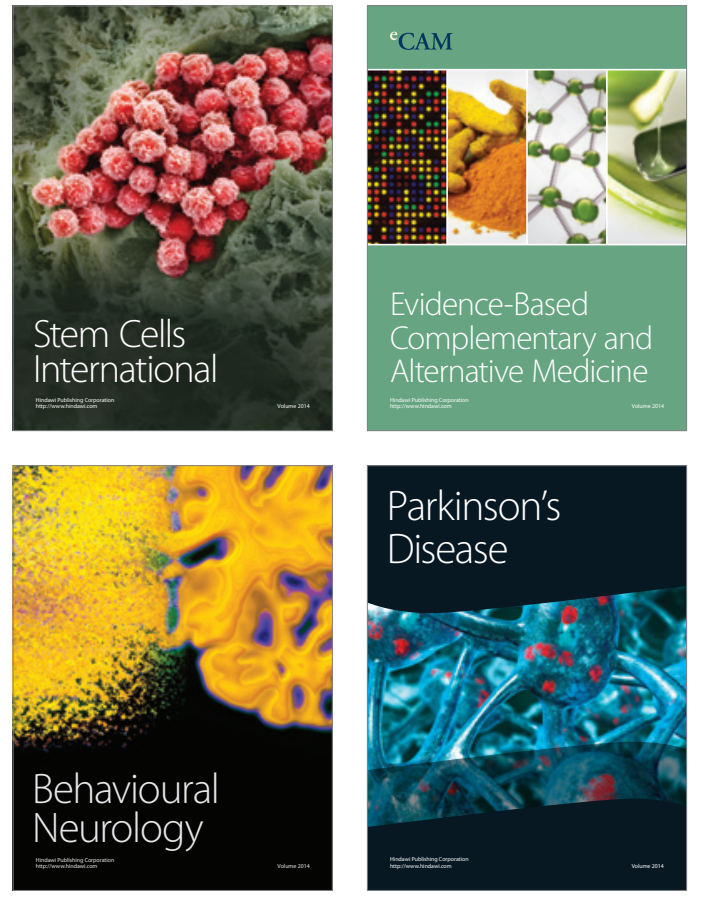
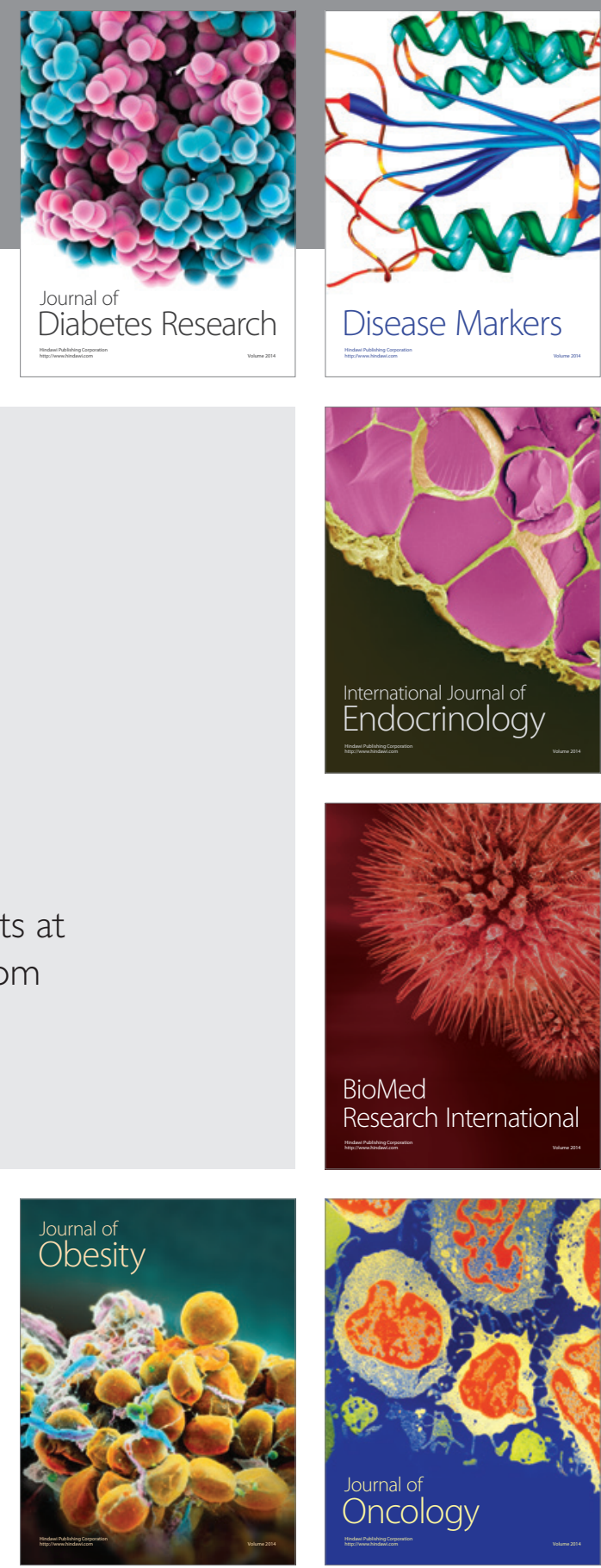

Disease Markers
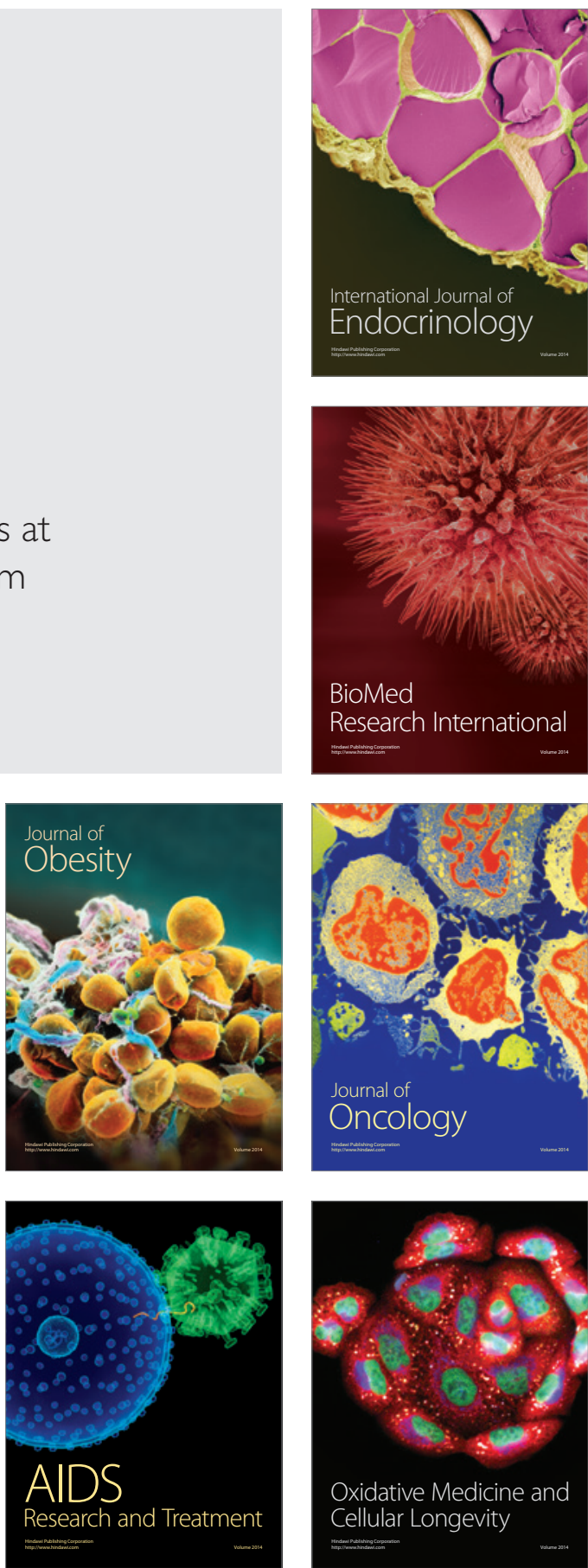